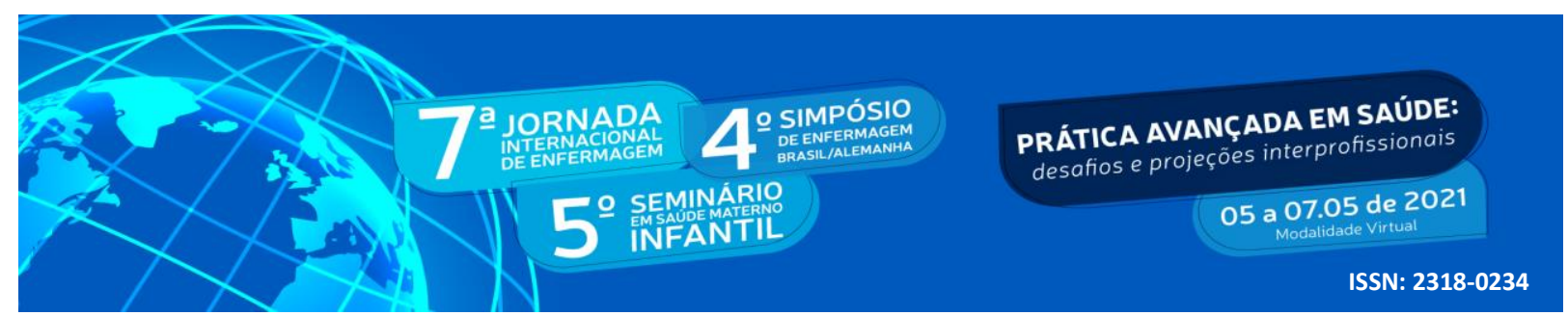

DOI: http://doi.org/10.48195/jie2021-016

\title{
PREVALÊNCIA E FATORES RELACIONADOS À LESÃO POR PRESSÃO EM IDOSOS
}

\section{Isabel Pires Barra'; Maria Alzira Rego Pinheiro²; Júlia Silva Fonseca dos Anjos³; Rafael Moreira do Nascimento ${ }^{4}$; Ana Elza Oliveira de Mendonça ${ }^{5}$.}

\begin{abstract}
RESUMO
Objetivou-se descrever a prevalência de lesões por pressão e fatores relacionados em pessoas idosas. Trata-se de uma revisão integrativa da literatura, realizada em janeiro de 2021 na Literatura LatinoAmericana e do Caribe de informação em Ciências da Saúde, Medical Literacture Analysis and Retrieval System Online, Bases de Dados de Enfermagem, U. S. National Library of Medicine, Índice Bibliográfico Espanhol de Ciências de Saúde e Scientific Electronic Library Online. Foram selecionados quatro artigos completos, publicados entre 2016 e 2019. A prevalência de lesão por pressão em idosos variou de 5,0\% a 23,0\%. Os fatores relacionados à sua ocorrência foram a idade avançada, presença de doenças crônicas não transmissíveis, restrições alimentares por condições socioeconômicas e mobilidade física prejudicada. Esses achados, denotam a importância da realização da educação permanente dos profissionais de saúde, com ênfase na investigação precoce dos fatores de risco, planejamento e implantação de estratégias de prevenção adequadas as necessidades individuais.
\end{abstract}

Palavras-chave: Lesão por pressão; Idoso; Enfermagem.

\begin{abstract}
The objective was to describe the prevalence of pressure injuries and related factors in the elderly. This is an integrative literature review, carried out in January 2021 in the Latin American and Caribbean Literature of Health Sciences information, Medical Literacture Analysis and Retrieval System Online, Nursing Databases, US National Library of Medicine, Spanish Bibliographic Index of Health Sciences and Scientific Electronic Library Online. Four complete articles were published, published between 2016 and 2019. The prevalence of pressure injuries in the elderly ranged from $5.0 \%$ to $23.0 \%$. The factors related to its occurrence were advanced age, presence of chronic non-communicable diseases, dietary restrictions due to socioeconomic conditions and impaired physical mobility. These findings denote the importance of conducting permanent education for health professionals, with an emphasis on early investigation of risk factors, planning and implementation of prevention strategies appropriate to individual needs.
\end{abstract}

\footnotetext{
1 Estudante do Curso de Enfermagem da Universidade Federal do Rio Grande do Norte E-mail: barraisa20@gmail.com;

${ }^{2}$ Enfermeira. Universidade Federal do Rio Grande do Norte. E-mail: mariaalzirarp@ gmail.com

3 Estudante do Curso de Enfermagem da Universidade Federal do Rio Grande do Norte. E-mail: julia.sanjos18@gmail.com;

${ }^{4}$ Mestrando do Programa de Pós-graduação em Enfermagem da Universidade Federal do Rio Grande do Norte. E-mail: rafhelmoreira@ hotmail.com

5 Orientadora. Doutora em Ciências da Saúde. Universidade Federal do Rio Grande do Norte. E-mail: anaelzaufrn@gmail.com
} 


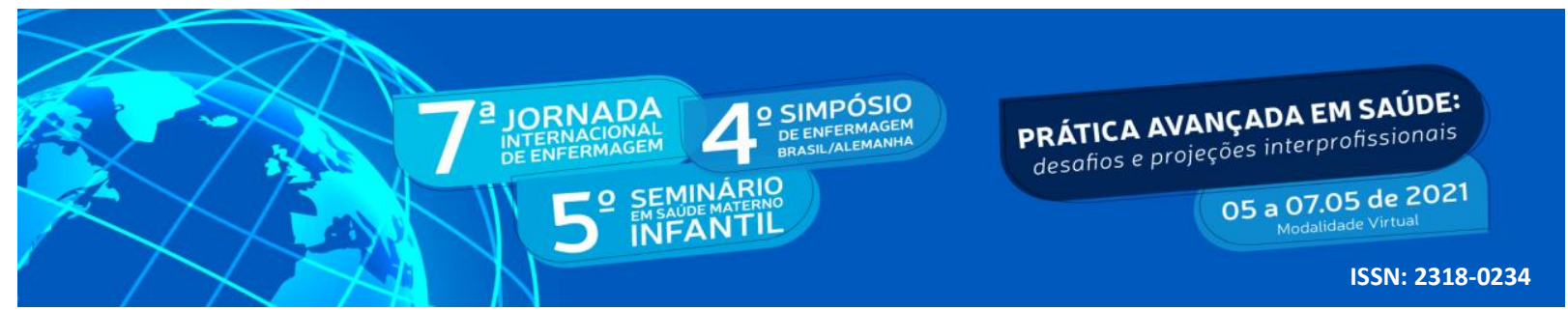

Key Words: Pressure ulcer; Aged; Nursing.

\section{INTRODUÇÃO}

As lesões por pressão (LP) são agravos cutâneos localizados, geralmente, sobre uma proeminência óssea, decorrentes da pressão excessiva, cisalhamento, além do uso de dispositivo médico ou outros artefatos (ALENCAR et. al., 2018; PONTES et al., 2020). Essa pressão constante nos tecidos diminui o fluxo sanguíneo, e consequentemente os níveis de oxigênio e nutrientes levando a perda da integridade cutânea (SANTOS et. al., 2016).

A LP é mais comum em idosos devido ao processo de envelhecimento, responsável por alterar a pele, tornando-a mais frágil e suscetível à pressão, atrito e cisalhamento. Outro aspecto importante é a limitação física e sensorial associadas à perda da capacidade de reposicionamento e às reduções de massa muscular e nível de albumina sérica. No entanto, uma assistência efetiva, individualizada, centrada no bem-estar e conforto do paciente pode minimizar seus efeitos (SANTOS et al., 2020).

O aumento exponencial de pessoas idosas, ou seja, com sessenta anos ou mais, é um fenômeno mundial. Este advento eleva a necessidade de hospitalização por enfermidades crônicas não transmissíveis, dentre as quais as LP, se constituem como uma síndrome geriátrica (PONTES et al., 2020). Além disso, as LP aumentam o tempo de permanência nos serviços de saúde, gerando aumento do consumo de materiais, medicamentos e demanda de cuidados pelos profissionais de saúde, especialmente da equipe de Enfermagem.

Adicionalmente aos custos, o surgimento de LP traz sofrimento físico, emocional e psíquico à pessoa idosa e aos seus familiares. A limitação da autonomia funcional pode também predispor a infecções graves, dores, ocorrência de amputações, que se traduzem em impacto negativo na qualidade de vida desses pacientes e de seus familiares (SARDELI et al., 2021).

Diante deste contexto, torna-se necessário conhecer a prevalência e os fatores associados à LP em idosos, para conhecer o perfil dessa população e elaborar estratégias de prevenção e promoção da saúde, como também para ações de recuperação, controle e tratamento, considerando as necessidades destes pacientes. 


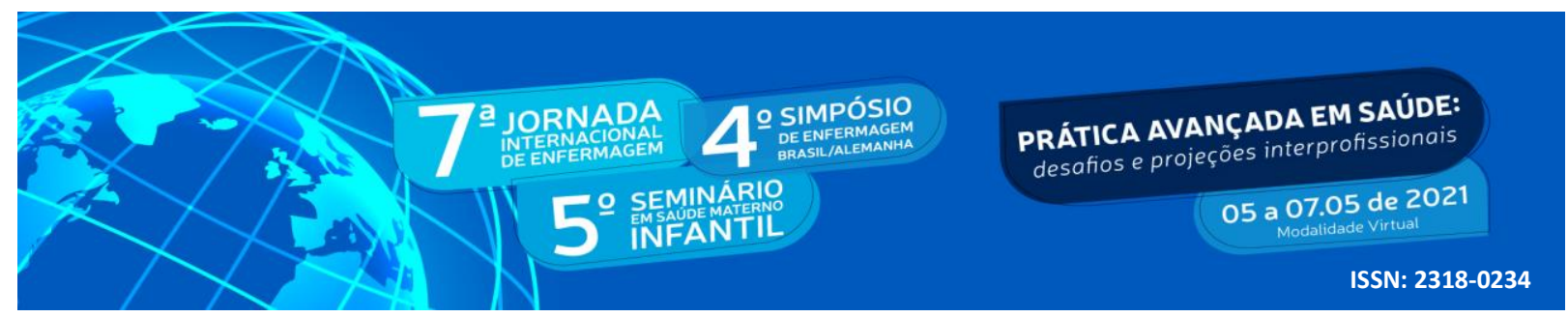

\section{OBJETIVO}

Descrever a prevalência de lesões por pressão e fatores relacionados à sua ocorrência em pessoas idosas.

\section{METODOLOGIA}

Trata-se de um estudo de revisão integrativa da literatura. Os estudos desenvolvidos com esse método reúnem e sintetizam resultados de pesquisas sobre um delimitado tema, de maneira sistemática e organizada, contribuindo com o aprofundamento do conhecimento do tema investigado (RAMALHO NETO et al., 2016).

Para a sua realização, seguiram-se as etapas: elaboração da pergunta norteadora; estabelecimento dos objetivos e critérios de seleção dos artigos; definição das informações a serem extraídas; seleção dos artigos; análise dos resultados; discussão dos achados (RAMALHO NETO et al., 2016).

As bases eletrônicas foram a Literatura Latino-Americana e do Caribe de informação em Ciências da Saúde (LILACS), Medical Literacture Analysis and Retrieval System Online (MEDLINE), Bases de Dados de Enfermagem (BDENF), U. S. National Library of Medicine (PUBMED), Índice Bibliográfico Espanhol de Ciências de Saúde (IBECS) e Scientific Electronic Library Online (SciELO). A busca em diversas bases de dados teve como finalidade ampliar o âmbito da pesquisa e minimizar possíveis vieses.

Foi elaborada a seguinte questão norteadora: “Qual a produção científica acerca da temática Lesão por pressão em idosos?". Para o levantamento das publicações, foram utilizados os descritores cadastrados nos Descritores em Ciências da Saúde (DeCS): "Lesão por pressão", "Idoso" e "Enfermagem"; "Pressure ulcer", "Aged" e "Nursing". Os cruzamentos foram feitos por meio do moderador booleano "AND” entre os descritores, utilizando o formulário para busca avançada. Essa etapa foi realizada no mês de janeiro de 2021.

Para refinar as buscas foram estabelecidos os seguintes critérios de inclusão: artigos originais, desenvolvidos com pessoas idosas, disponíveis no formato texto completo, nos idiomas inglês, português e espanhol, publicados no período entre 2016 a 2021. Os critérios de 


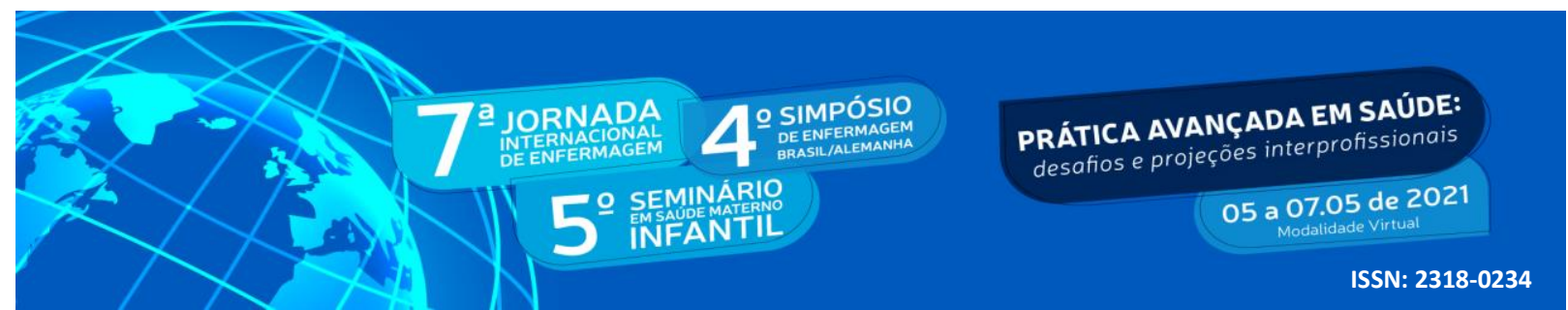

exclusão adotados foram: artigos que não contribuíssem para responder ao questionamento proposto, dissertações, teses, estudos em formato de editorial e carta ao editor.

A partir da busca realizada nas fontes de dados e após o refinamento, foram encontrados 355 artigos científicos, sendo 5 na SciELO, 25 na LILACS, 120 da MEDLINE, 164 na PUBMED, 12 no IBECS e 29 na BDENF. A seleção dos artigos foi realizada inicialmente por meio da leitura de títulos e resumos de todos os artigos. Foram selecionados 12 artigos.

Após, a leitura minuciosa dos 12 artigos que atenderam aos critérios de seleção, foram excluídas oito publicações, que não contribuíam efetivamente para elucidar a questão norteadora da presente pesquisa. Assim, a amostra final foi composta por quatro artigos. Os autores utilizados neste estudo foram devidamente referenciados, respeitando e identificando adequadamente as fontes de pesquisa, em atenção ao rigor ético quanto à propriedade intelectual dos textos científicos.

\section{RESULTADOS E DISCUSSÃO}

Na presente revisão, após os critérios de inclusão e exclusão, analisaram-se quatro artigos científicos cujos resultados permeiam os objetivos do presente estudo. Os artigos selecionados foram organizados de acordo com as seguintes variáveis: autores, ano de publicação, título, periódico e banco de dados, dispostos no Quadro 1.

Quadro 1 - Distribuição dos artigos selecionados segundo autor, ano, título, periódico e fonte de dados. Brasil, Natal/RN, 2021.

\begin{tabular}{|c|c|c|c|c|}
\hline $\mathbf{N}^{\circ}$ & $\begin{array}{c}\text { AUTORES/ } \\
\text { ANO }\end{array}$ & TÍTULO & PERIÓDICO & FONTE \\
\hline 1 & $\begin{array}{l}\text { CAVALCAN } \\
\text { TE et al, } \\
2016 \text {. }\end{array}$ & $\begin{array}{l}\text { Indicadores de saúde e a } \\
\text { segurança do idoso } \\
\text { institucionalizado }\end{array}$ & $\begin{array}{l}\text { Rev. Esc. } \\
\text { Enferm. USP }\end{array}$ & $\begin{array}{l}\text { SciELO, } \\
\text { LILACS, Bdenf e } \\
\text { Medline }\end{array}$ \\
\hline
\end{tabular}




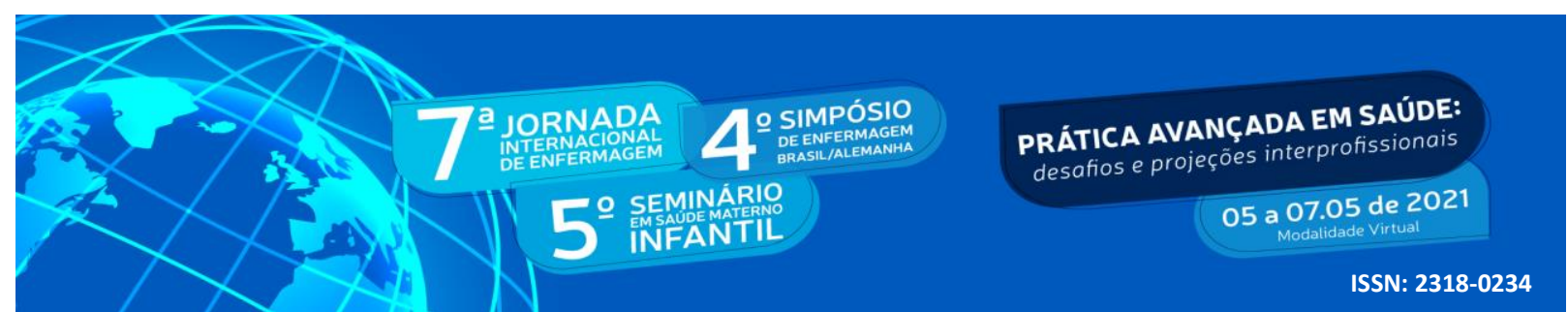

\begin{tabular}{|c|l|l|l|l|}
\hline 2 & VIEIRA; & $\begin{array}{l}\text { Prevalência e fatores associados } \\
\text { ARAÚJO, } \\
2018 .\end{array}$ & $\begin{array}{l}\text { Rev. Esc. } \\
\text { a feridas crônicas em idosos na } \\
\text { atenção básica }\end{array}$ & SciELO \\
\hline 3 & $\begin{array}{l}\text { VIEIRA et al, USP } \\
2018 .\end{array}$ & $\begin{array}{l}\text { Risco de lesão por pressão em } \\
\text { idosos com comprometimento } \\
\text { na realização de atividades } \\
\text { diárias }\end{array}$ & $\begin{array}{l}\text { Rev. Enferm. } \\
\text { Centro-Oeste } \\
\text { Mineiro }\end{array}$ & LILACS e Bdenf \\
\hline 4 & $\begin{array}{l}\text { SARI et al, } \\
2019 .\end{array}$ & $\begin{array}{l}\text { The prevalence of pressure } \\
\text { ulcers in community-dwelling } \\
\text { olderadults: A study in an } \\
\text { Indonesian city }\end{array}$ & Pound J. & PubMed \\
\hline
\end{tabular}

Fonte: dados da pesquisa, 2021.

Identificou-se que os desenhos metodológicos usados pelos autores foram: três estudos do tipo transversal analítico e descritivo e um estudo documental retrospectivo. Quanto ao local de realização, três foram realizados no Brasil e um na Indonésia, todos publicados entre os anos de 2016 e 2019.

As populações das amostras foram compostas por no mínimo de 42 e no máximo de 339 pacientes idosos, com médias de idades entre 65 e 80 anos, em relação ao gênero em todos os estudos prevaleceu o sexo feminino. Em três estudos os participantes estavam em seus domicílios e em um os idosos estavam em Instituições de Longa Permanência. A maioria dos estudos não traz informações sobre os dados clínicos de saúde e comorbidades dos envolvidos na pesquisa.

A predominância de idosos do sexo feminino nos estudos brasileiros, pode ser reflexo do aumento da expectativa de vida da população feminina. De acordo com o censo do IBGE realizado em 2010 as mulheres representavam 55,5\% da população idosa brasileira e 61,0\% eram idosas com mais de 80 anos de idade (IBGE, 2010).

Cavalcante et al. (2016), realizaram um estudo com 233 idosos e identificaram alta prevalência de lesão por pressão $(23,0 \%)$, quando comparado com outra pesquisa dessa revisão 


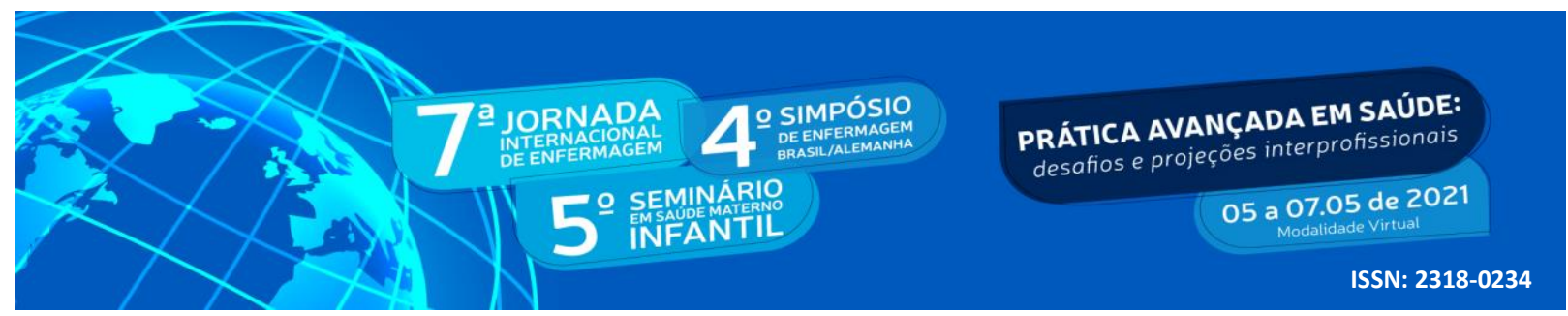

realizada com 235 idosos residentes em uma cidade da Indonésia, no qual a prevalência foi de 10,8\% (SARI et al., 2019). Identificar a prevalência de lesão por pressão em idosos é um importante indicador a ser mensurado para avaliar a qualidade de vida e de saúde da população.

Vieira e Araújo (2018), identificaram a menor prevalência de LP em idosos, dentre os artigos analisados, ou seja, em uma amostra de 339 a prevalência foi de 5,0\%. É importante destacar que os participantes do estudo residiam em seus domicílios e eram acompanhadas pelas equipes da Estratégia Saúde da Família (VIEIRA; ARAÚJO, 2018).

Os fatores relacionados ao surgimento das lesões por pressão identificados nos estudos analisados, que se destacaram foram: idade avançada, presença de doenças crônicas não transmissíveis, restrições alimentares por condições socioeconômicas favorecendo à desnutrição, déficit cognitivo, mobilidade física prejudicada ou imobilização física e dificuldade para realizar Atividades de Vida Diárias (AVD).

$\mathrm{Na}$ assistência de enfermagem ao paciente idoso com o risco de desenvolver LP, é de suma importância o uso de métodos de avaliação do estado clínico do paciente, atrelado aos fatores ambientais e organização da equipe. Dentre as mais utilizadas para o processo de mensuração do risco de LP, identificou-se a Escala de Braden (Braden Scale), que busca associar os fatores ambientais e clínicos que podem levar ao surgimento das lesões (VIEIRA; ARAÚJO, 2018; VIEIRA et al., 2018).

Outra importante forma de avaliação precoce do risco de desenvolvimento de lesões nos pacientes idosos, devido ao avançar da idade e diminuição da mobilidade, é a Escala de Katz, a qual avalia o nível de independência dos mesmos relacionados às atividades de vida diárias (AVD). Por meio desta escala, é possível determinar o nível de dependência para a realização das AVD, e quanto a dependência maior é a predisposição ao aparecimento de lesões devido a redução da mobilidade física (VIEIRA; ARAÚJO, 2018; VIEIRA et al., 2018).

De acordo com Vieira e Araújo (2018), idosos que não desenvolvem nenhuma atividade física possuem maiores chances de desenvolver LP, já ter mobilidade ativa e não ter restrições alimentares favorecem uma associação negativa em relação ao surgimento dessas lesões (VIEIRA; ARAÚJO, 2018).

No estudo desenvolvido por Vieira et al. (2018) com 42 idosos institucionalizados, após aplicação da escala de Katz, foi observado que 100,0\% dos participantes apresentaram dependência para atividades simples, como tomar banho, vestir-se, usar o vaso sanitário e 


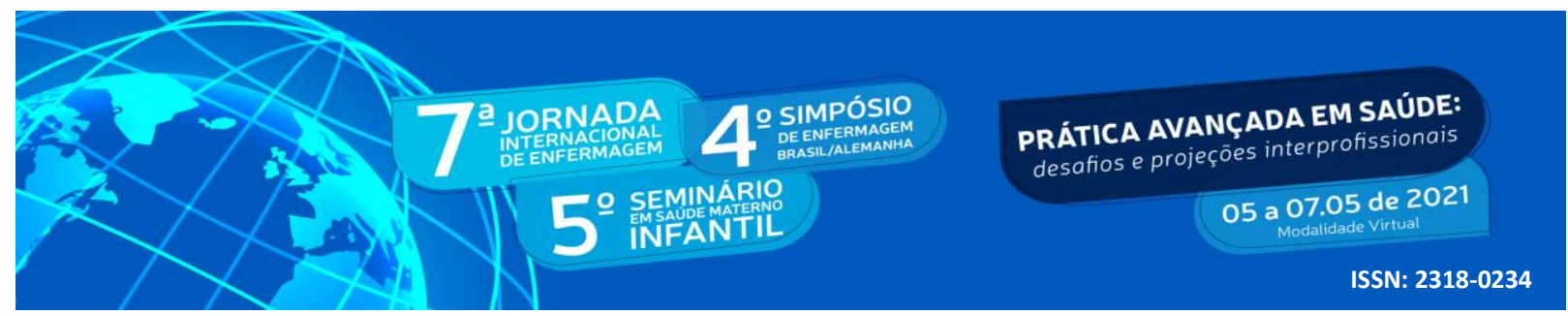

transferir-se de um local para o outro. Os autores reforçam que ter maior grau de dependência para realizar as AVD, significa maior o risco de desenvolver LP (VIEIRA et al., 2018).

A mobilidade dos idosos está diretamente atrelada ao maior risco de desenvolvimento das lesões por pressão. Cerca de 64,3\% idosos institucionalizados foram identificados após análise por meio da escala de Braden, com risco de desenvolver lesões, dentre os quais 50\% encontravam-se em risco moderado a alto, por sua maior dependência para realização das atividades de vida diária (VIEIRA et al., 2018).

Em uma Instituição de Longa Permanência, foram identificados déficits que impactaram na implementação de ações para a prevenção do surgimento de lesões por pressão. Entre eles, o baixo quantitativo de profissionais de enfermagem na assistência de idosos com alto grau de dependência nas atividades de vida diárias (AVD). Além disso, a desnutrição, a dificuldade na implantação de protocolos voltados à classificação e tratamento das lesões, o uso precário de materiais de higiene pessoal, somado a falta de capacitação para o cuidado continuado enfraquecem as condições de prevenção ao dano tecidual (CAVALCANTE et al., 2016).

Com enfoque nas ações de prevenção de LP, pesquisadores canadenses propuseram um modelo organizacional para facilitar denominado Factors Facilitating Pressure Ulcer Prevention Model (FFPUPM). O modelo descreve como deve ser a distribuição da equipe de saúde em diferentes instâncias do serviço, com ênfase na cultura organizacional da prevenção de LP e melhoria contínua da qualidade (STADNYK et al., 2018).

O FFPUPM inclui tópicos como: conselho de liderança composto por profissionais de enfermagem e equipe médica, responsáveis pela capacitação dos demais profissionais e organização da rotina de cuidados voltados à prevenção de lesões; educação continuada, assim como educação da condição clínica do paciente com os familiares; melhoria contínua da qualidade, associada a uma prática clínica baseada em evidências e campeões em nível de unidade que compõe o conselho de liderança no contexto da prevenção de LP (STADNYK et al., 2018).

Vale ressaltar que as medidas de prevenção são muito importantes, contudo, não basta apenas aplicar as escalas no intuito de avaliar o risco de lesões. Após identificar o risco de LP o enfermeiro deve planejar e prescrever quais ações preventivas devem ser implementadas pelos familiares e cuidadores, com o objetivo comum de não apenas prevenir a lesão por pressão, como também promover a saúde e qualidade de vida das pessoas idosas. 


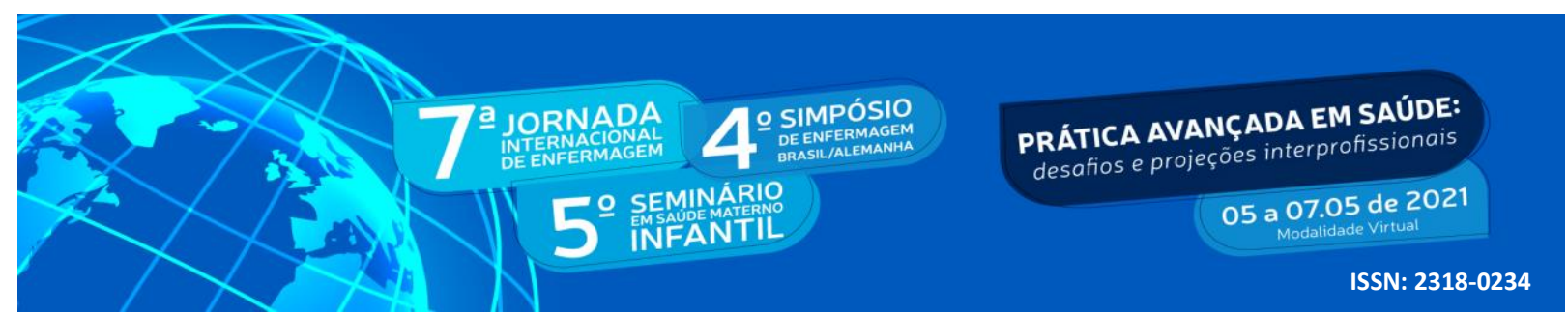

\section{CONCLUSÃO}

Os achados do presente estudo apontam que a prevalência de Lesão por Pressão é elevada em idosos, especialmente naqueles com limitação e mobilidade física prejudicada. Os fatores relacionados ao desenvolvimento de lesões foram a idade avançada, presença de doenças crônicas não transmissíveis, restrições alimentares por condições socioeconômicas e mobilidade física prejudicada.

Os dados apresentados e discutidos reforçam o papel dos aspectos clínicos e socioeconômicos na prevalência de Lesão por Pressão. Adicionalmente, destaca-se a importância da realização de atividades de educação permanente com os profissionais de saúde e com os familiares e cuidadores.

O foco das ações educativas deve ser a investigação precoce dos fatores de risco, a elaboração de estratégias de prevenção e o tratamento, no intuito de aperfeiçoar os cuidados, assegurar o monitoramento de eventos adversos e contribuir para a qualidade de vida dos idosos.

\section{REFERÊNCIAS}

ALENCAR, G. S. A. et al. Lesão por pressão na unidade de terapia intensiva: incidência e fatores de riscos. Rev Nursing, 2018. Disponível em:

http://www.revistanursing.com.br/revistas/239-

Abril2018/lesao_por_pressao_na_unidade_de_terapia_intensiva.pdf. Acesso em 28 fev. 2021.

CAVALCANTE, M. L. S. N. et al. Indicadores de saúde e a segurança do idoso institucionalizado. Rev. esc. enferm. USP, São Paulo, v. 50, n. 4, p. 602-609, ago. 2016.

Disponível em: http://www.scielo.br/scielo.php?script=sci_arttext\&pid=S008062342016000400602\&lng=pt\&nrm=iso . Acesso em 14 mar. 2021.

IBGE - INSTITUTO BRASILEIRO DE GEOGRAFIA E ESTATÍSTICA. Censo Brasileiro de 2010. Rio de Janeiro: IBGE, 2011. Disponível em: https://censo2010.ibge.gov.br/ . Acesso em: 12 mar. 2021.

PONTES, I. E. A. et al. Associated factors with pressure injury in elderly people with gastrointestinal cancer. Rev. Research, Society and Development, v. 9, n. 7, 2020. Disponível em: https://rsdjournal.org/index.php/rsd/article/view/4279/4251 Acesso em $28 \mathrm{fev}$ 2021. 


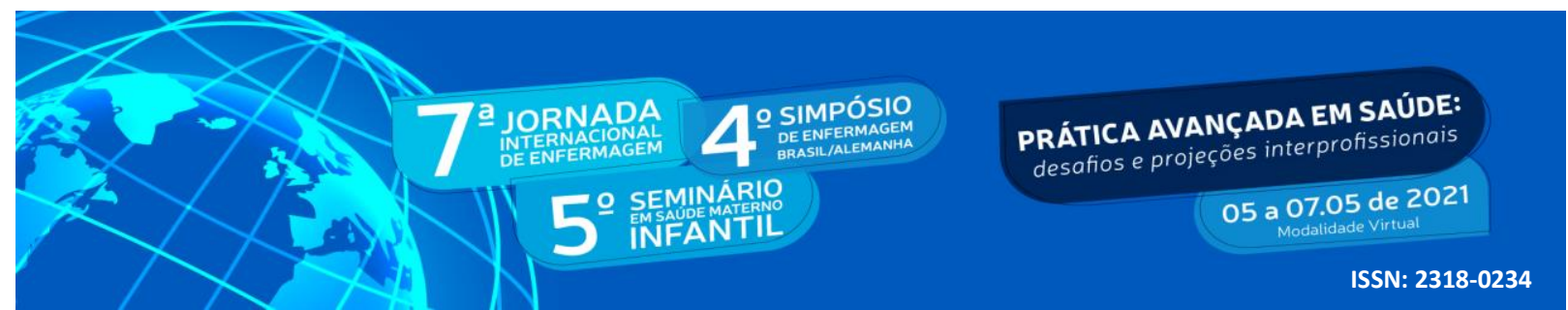

SANTOS, C.T. et al. Diagnóstico de enfermagem risco de úlcera por pressão: validação de conteúdo. Rev Latino-Americana de Enfermagem,2016. Disponível em:

http://www.scielo.br/pdf/rlae/v24/pt_0104-1169-rlae-24-02693.pdf. Acesso em 28 fev 2021.

SANTOS, W. P. et al. Prevention of pressure injury in elderly house hold imobility. Rev. Brazilian Journal of Development, v. 6, n. 10, 2020. Disponível em:

https://www.brazilianjournals.com/index.php/BRJD/article/view/19075/15324. Acesso em 28 fev 2021.

SARDELI, K. M. et al. Pressure injury in long-term institutions for the elderly: an integrative review. Rev. Brazilian Journal of Development, v. 7, n. 2, 2021. Disponível em:

https://www.brazilianjournals.com/index.php/BRJD/article/view/24143/19328. Acesso em 28 fev 2021.

SARI, S. P. et al. The prevalence of pressure ulcers in community-dwelling olderadults: A study in an Indonesian city. Int Wound J., v. 16, p. 534-541, 2019. Disponível em: https://onlinelibrary.wiley.com/doi/epdf/10.1111/iwj.13081. Acesso em 14 mar. 2021.

STADNYK, B. et al. Factors in facilitating an organisational culture to prevent pressure ulcers among older adults in health-care facilities. J Wound Care., v. 1, e. 27(Sup7):S4-S10, 2018. Available from:

https://www.magonlinelibrary.com/doi/pdfplus/10.12968/jowc.2018.27.Sup7.S4 . Access on em 14 Mar. 2021.

VIEIRA, C. P. B.; ARAUJO, T. M. E. Prevalência e fatores associados a feridas crônicas em idosos na atenção básica. Rev. esc. enferm. USP, São Paulo, v. 52, e. 03415, 2018.

Disponível em: http://www.scielo.br/scielo.php?script=sci_arttext\&pid=S0080-

62342018000100491\&lng=pt\&nrm=iso. Acesso em 14 mar. 2021.

VIEIRA, V. A. S. et al. Risco de lesão por pressão em idosos com comprometimento na realização de atividades diárias. Revista de Enfermagem do Centro-Oeste Mineiro, v. 8, e. 2599, 2018. Disponível em: http://seer.ufsj.edu.br/index.php/recom/article/view/2599/1940. Acesso em 14 mar. 2021. 